\title{
A molecularly imprinted polymer-based evanescent wave fiber optic sensor for the detection of basic red 9 dye
}

\author{
Marcos V. Foguel ${ }^{a}{ }^{*}$, Xuan-Anh Ton $^{\mathrm{b}}$, Maria V.B. Zanoni ${ }^{\mathrm{a}}$, Maria Del Pilar T. Sotomayor ${ }^{\mathrm{a}}$, \\ Karsten Haupt ${ }^{\mathrm{b}}$, Bernadette Tse Sum Bui ${ }^{\mathrm{b}, * *}$ \\ a Institute of Chemistry, Unesp-Univ Estadual Paulista, Rua Francisco Degni, 55, 14.800-060 Araraquara, Brazil \\ b Sorbonne Universités, Université de Technologie de Compiègne, CNRS Laboratory for Enzyme and Cell Engineering, CS60319, 60203 Compiègne cedex, \\ France
}

\section{A R T I C L E I N F O}

\section{Article history:}

Received 17 December 2014

Received in revised form 4 May 2015

Accepted 6 May 2015

Available online 14 May 2015

\section{Keywords:}

Molecularly imprinted polymer

Evanescent wave fiber optic sensor

Textile dye

Basic red 9

\begin{abstract}
A B S T R A C T
A cheap and robust method for the detection and quantification of textile dyes is the use of biomimetic sensors with optical transduction, employing a molecularly imprinted polymer (MIP) as the recognition element. This paper presents the optimization of a MIP for the dye, basic red 9 (BR9) and the immobilization of these polymers on a disposable $4-\mathrm{cm}$ long polystyrene optical waveguide for the development of an evanescent wave fiber optic sensor aimed at the determination and quantification of this dye in different matrices and industrial effluents. MIPs were synthesized using 2-acrylamido-2-methyl1-propanesulfonic acid and ethylene glycol dimethacrylate, as functional monomer and cross-linker, respectively. The polymer was then immobilized on the waveguide by dip-coating the fiber in the polymer suspension. The sensing element was interrogated with a fiber-coupled spectrophotometer. BR9 could be detected in the low $\mu \mathrm{M}$ range, thus making it a promising device for determining this compound in textile effluents.
\end{abstract}

(C) 2015 Elsevier B.V. All rights reserved.

\section{Introduction}

Molecularly imprinted polymers (MIPs) are biomimetic synthetic receptors possessing specific cavities designed for a target molecule. Produced by a templating process at the molecular level by co-polymerization of functional and cross-linking monomers, MIPs are capable of recognizing and binding target molecules with specificities and affinities comparable to those of natural receptors [1-3]. These tailor-made synthetic receptors have considerable advantages over biological recognition materials such as enzymes and antibodies, as they are more easily prepared, cheaper and are resistant to adverse conditions such as the presence of acids, bases, organic solvents, high temperatures and high pressure. Due to these advantages, they have become serious alternatives as recognition elements in biosensors [4-8]. In particular, MIPs have been widely used in optical chemical sensors, also known as optodes. The optodes are very often based on optical fibers where the MIP is immobilized on the surface [6,9]. The applications of optical

\footnotetext{
* Corresponding author. Tel.: +55 16 33019740; fax: +55 1633222308

** Corresponding author. Tel.: +33 344234402; fax: +33 344203910.

E-mail addresses: mvfoguel@iq.unesp.br (M.V. Foguel), jeanne.tse-sum-bui@utc.fr (B. Tse Sum Bui).
}

fibers as sensors permeated several fields of great importance in analytical chemistry, such as environmental, food, chemical and clinical analyses $[10,11]$. Optodes present some advantages, including simple manufacturing, low cost, good selectivity and sensitivity, real-time and remote analysis possibility and allow the development of "disposable" sensors, as well as easy replacement. From the environmental point of view, optodes can be used for the detection of toxic compounds released in effluents; among these pollutants include dyes. In the textile industry, $\sim 50 \%$ of the dyes are lost in the dyeing process and $\sim 10-15 \%$ are discarded in wastewater, causing considerable degradation of the environment by modifying the natural color and forming foams on the surface [12].

Dyes are classified according to their chemical structures and their applications. These compounds consist of chromophores, responsible for the dye coloration $[13,14]$. The dyes are classified as azo, anthraquinone, triphenylmethane and heterocyclic dyes [12]. Among these dyes, triphenylmethanes are widely used in the textile industry for the coloration of nylon, wool, silk and cotton and represent a major environmental concern due to its potential toxicity to animals and humans [15]. In effluents, these products cause environmental problems, because they absorb light and interfere with aquatic biological processes [16]. Moreover these toxic compounds are not eliminated by conventional wastewater treatment plants. 
<smiles>Nc1ccc(C(=C2C=CC(=[NH2+])C=C2)c2ccc(N)cc2)cc1</smiles>

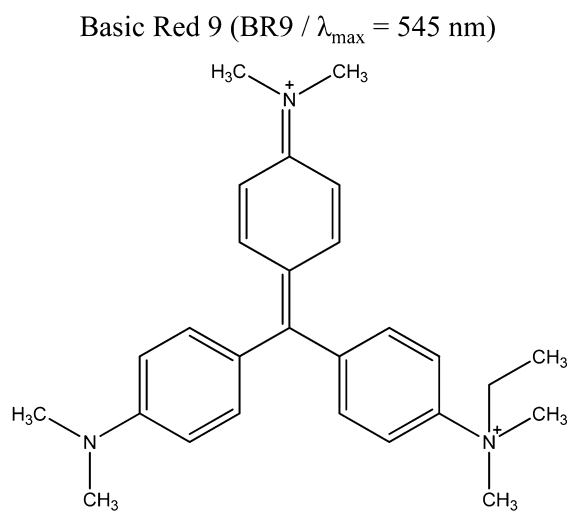

Methyl Green $\left(\mathrm{MG} / \lambda_{\max }=636 \mathrm{~nm}\right)$<smiles>[R][M]OC([R])=[N+]([R])[O-]</smiles>

Acid green $16\left(\mathrm{AG} 16 / \lambda_{\max }=642 \mathrm{~nm}\right)$<smiles>CC(=O)Nc1cc(S(=O)(=O)[O-])cc2cc(S(=O)(=O)[O-])c(N=Nc3ccccc3)c(O)c12</smiles>

Acid Red $1\left(\mathrm{AR} 1 / \lambda_{\max }=536 \mathrm{~nm}\right)$

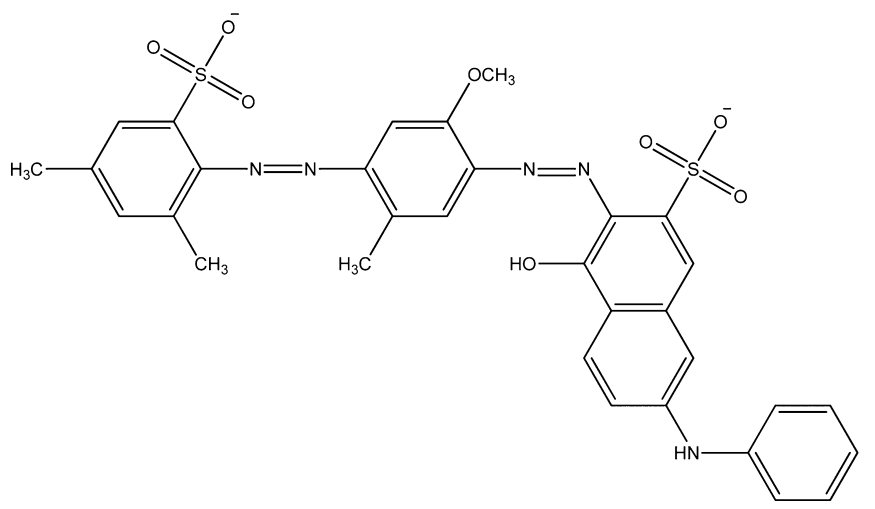

Direct violet $51\left(\right.$ DV51 $\left./ \lambda_{\max }=552 \mathrm{~nm}\right)$

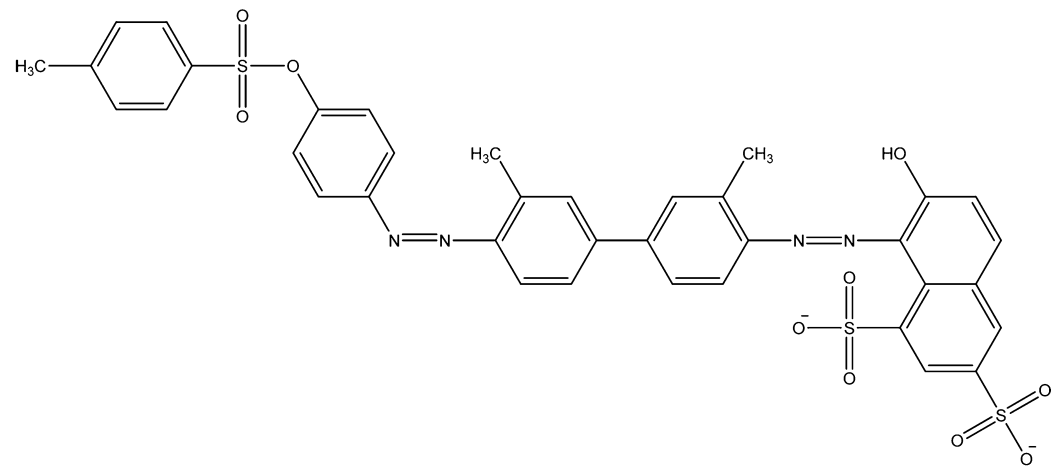

Acid red $114\left(\mathrm{AR} 114 / \lambda_{\max }=526 \mathrm{~nm}\right)$<smiles>Cc1cc(NC(=O)Nc2ccc(N=Nc3cc(S(=O)(=O)[O-])c4cccc(S(=O)(=O)[O-])c4c3)c(C)c2)ccc1N=Nc1cc(S(=O)(=O)[O-])c2cccc(S(=O)(=O)[O-])c2c1</smiles>

Direct yellow $50\left(\right.$ DY50 / $\left.\lambda_{\max }=396 \mathrm{~nm}\right)$

Fig. 1. Chemical structures of the target dye, BR9 and other textile dyes used in this study. BR9 and MG are chlorides and the other dyes are sodium salts.

Recently, we have developed a disposable evanescent wave fiber optic sensor coated with a MIP on a 4-cm long injection-molded tapered polystyrene waveguide, as a selective fluorescence probe [17]. An evanescent wave sensor has the property of surface specific detection, as the molecules not bound to the MIP, will contribute very little to the measured signal, thus its advantage especially when working with complex samples. Normally, an evanescent field is generated when total internal reflection of light occurs in an 
optical fiber coated with a lower refractive index material. This field propagates perpendicularly to the fiber surface into the medium, decaying exponentially with distance [18-20]. In that work, the evanescent light was used to excite a fluorescent signaling moiety contained in the MIP. Fluorescence enhancement occurred upon analyte binding to the MIP with the fluorescence intensity being proportional to the analyte concentration.

In the present work, instead of detecting the return evanescent wave fluorescence, we measure the evanescent wave absorbance $[9,21,22]$ during binding of the textile dye, basic red 9 (BR9) to a MIP. BR9 is a strong absorbing dye at $545 \mathrm{~nm}$. If a chromophoric analyte binds to the MIP-coated layer, the evanescent wave is absorbed by the analyte and the unabsorbed light is coupled back into the fiber and can be measured. In other words, the more dye there is bound to the MIP, the more light will be absorbed, and the less light will go back through the fiber to get measured [21,22]. Basic red 9 monohydrochloride, also known as pararosaniline chloride or basic fuchsin (CI 42500) (Fig. 1), belongs to the class of triphenylmethane dyes and it is a dark green crystalline powder. It is known to be stable under normal temperature and pressure, but can decompose when heated. In the dyeing process, the dye is used to obtain magenta color [23]. There are indications that this compound presents carcinogenic activity in humans, based on studies that show carcinogenicity in animals $[24,25]$. Due to the high toxicity and serious environmental effects posed by this dye, detection and quantification of basic red 9 in effluents are of paramount importance. In the light of that, this work presents the results obtained for the synthesis and characterization of MIPs for BR9. In the aim to develop an evanescent wave absorbance fiber optic sensor for the detection and quantification of this dye in different matrices and industrial effluents, the MIPs were immobilized on the waveguide by dip-coating the fiber in the polymer suspension. The sensing element was then interrogated with a fiber-coupled spectrometer (Fig. 2B).

\section{Materials and methods}

\subsection{Reagents and materials}

All chemicals, dyes and solvents were of analytical grade and purchased from Sigma-Aldrich (St-Quentin Fallavier, France), unless otherwise stated. 2,2'-Azobis(2,4-dimethylvaleronitrile) (ABDV) was obtained from DuPont Chemicals (Wilmington, USA). The monomers were employed as received. Water was purified using a Milli-Q system (Millipore, Molsheim, France). The polystyrene optical waveguides were from Research International, Inc. (Monroe, Washington, USA). Absorbance measurements of BR9 dye were done on Uvikon XS Secomam spectrophotometer. The size and morphology of the polymers was characterized using field emission scanning electron microscopy using a JEOL JSM 6330F.

\subsection{MIP synthesis}

The synthesis was inspired from Andersson and Ramström [26]. The polymers were prepared by bulk polymerization in a glass vial fitted with an air-tight septum. Typically, $0.02 \mathrm{mmol}$ of BR9, $0.06 \mathrm{mmol}$ of 2-acrylamido-2-methyl-1-propanesulfonic acid (AMPSA), $4 \mathrm{mmol}$ of ethylene glycol dimethacrylate (EGDMA) and $0.06 \mathrm{mmol}$ of ABDV were dissolved together in $1.5 \mathrm{~mL}$ of methanol/water (4/1). The mixture was purged with nitrogen for $5 \mathrm{~min}$ and polymerization was done overnight at $40^{\circ} \mathrm{C}$ in a waterbath. The bulk polymers were ground manually with a mortar and pestle, transferred to $2 \mathrm{~mL}$ microcentrifuge tubes and milled with $2.8 \mathrm{~mm}$ ceramic beads in the presence of methanol in a Precellys 24 homogenizer (Bertin Technologies, Montigny-le-Bretonneux,
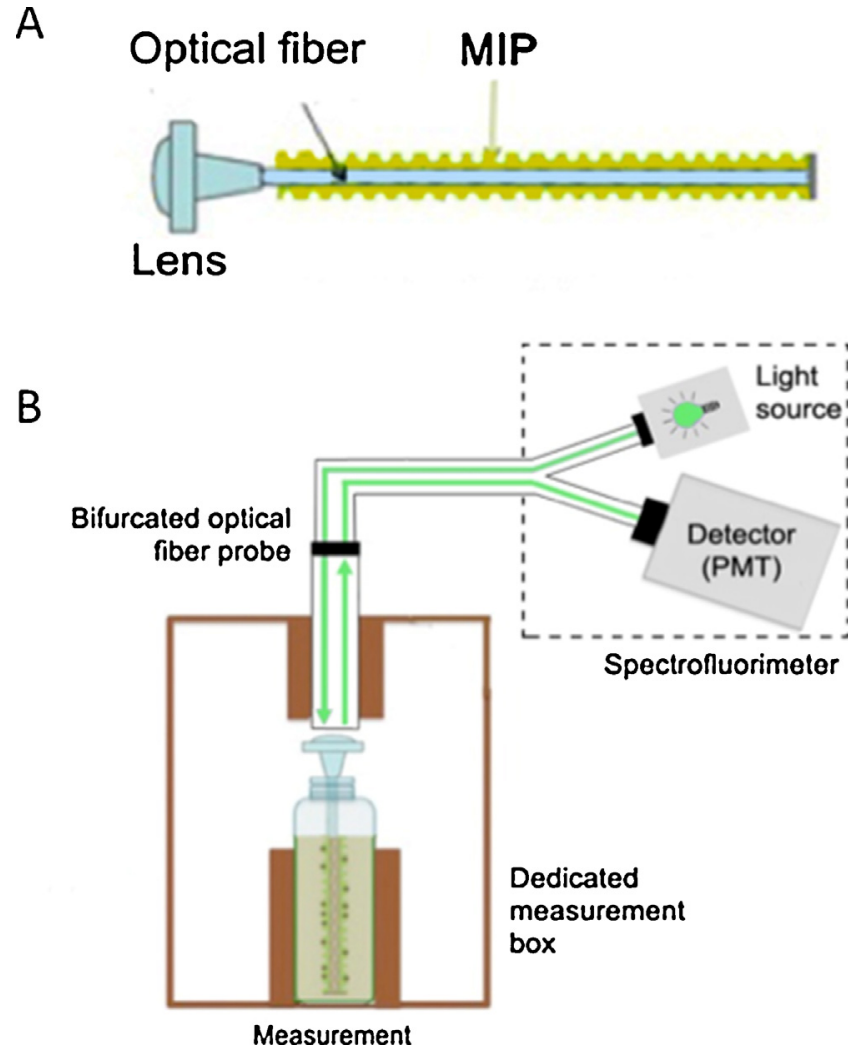

Fig. 2. (A) Drawing of the disposable 4-cm long polystyrene fiber optic waveguide coated with MIP. (B) Detection $(\lambda=545 \mathrm{~nm})$ of BR9 with the bifurcated optical fiber probe of a Horiba Fluorolog-3 spectrofluorimeter.

France). The polymers were transferred to $50 \mathrm{~mL}$ centrifuge tubes and washed at room temperature on a tube rotator (SB2, Stuart Scientific) with 3 rounds of methanol/acetic acid (7/3) and 3 rounds of methanol. They were then dried overnight under vacuum. Nonimprinted polymers (NIPs) were synthesized in the same way but without the addition of the imprinting template.

\subsection{Equilibrium binding studies of BR9 with the bulk polymers}

A stock solution of $100 \mu \mathrm{M}$ of BR9 was prepared in water and stored at $4{ }^{\circ} \mathrm{C}$. The polymer particles, at a concentration of $20 \mathrm{mg} / \mathrm{mL}$, were suspended by sonication in water. From this stock, various polymer concentrations $(1-8 \mathrm{mg} / \mathrm{mL})$ were pipetted in $1.5 \mathrm{~mL}$ polypropylene microcentrifuge tubes and $10 \mu \mathrm{M}$ of BR9 dye was added. The final volume was adjusted to $1 \mathrm{~mL}$. Different binding media were tested: water, $0.05 \% \mathrm{NH}_{3}(\mathrm{v} / \mathrm{v}), 1.0 \% \mathrm{NH}_{3}(\mathrm{v} / \mathrm{v})$ and $0.1 \mathrm{M}$ Britton-Robinson buffer solutions at $\mathrm{pH}$ 2.0, 5.0, 7.0 and 10.0. The tubes were incubated for $4 \mathrm{~h}$ at ambient temperature on a tube rotator, followed by centrifugation for $30 \mathrm{~min}$ at $30,000 \mathrm{~g}$. Then absorbance measurements were performed on the supernatants using a fixed wavelength of $545 \mathrm{~nm}$ or $242 \mathrm{~nm}$ (for incubation media containing ammonia, as the dye turns colorless). Calibration curves of BR9 at these two wavelengths (Figs. S1 and S2, SI) show the linear dependency of absorbance at the concentrations of dye used.

\subsection{Selectivity studies}

To evaluate the selectivity of the BR9 imprinted polymers, the equilibrium binding behaviors with six other dyes, Methyl Green (MG), Acid Green 16 (AG16), Acid Red 1 (AR1), Direct 
A

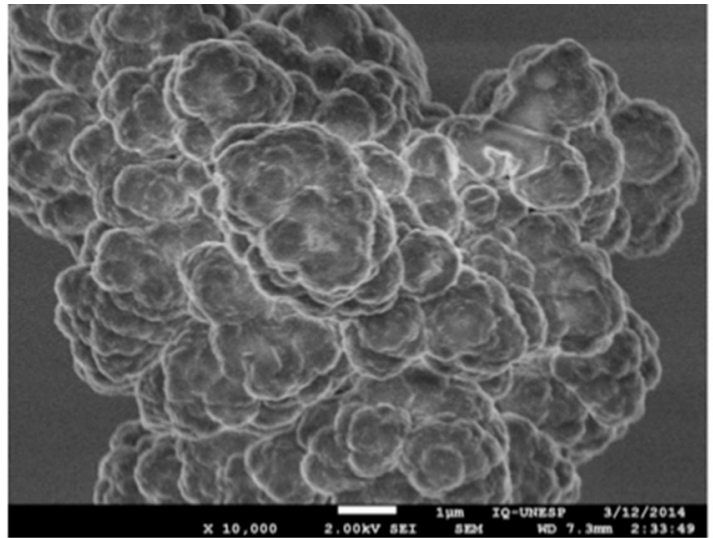

$\mathrm{B}$

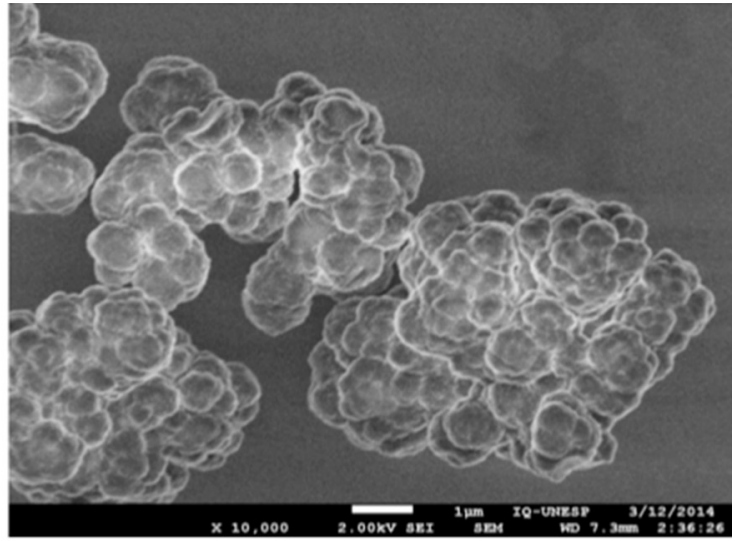

$\mathrm{C}$

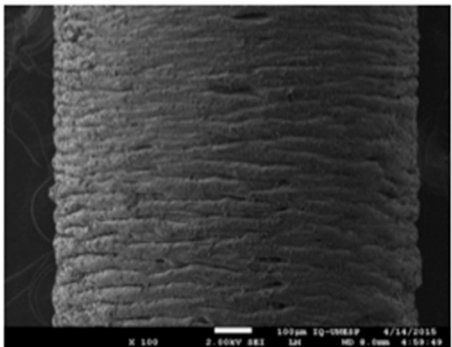

F

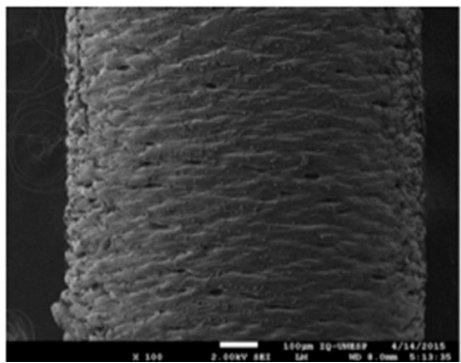

D

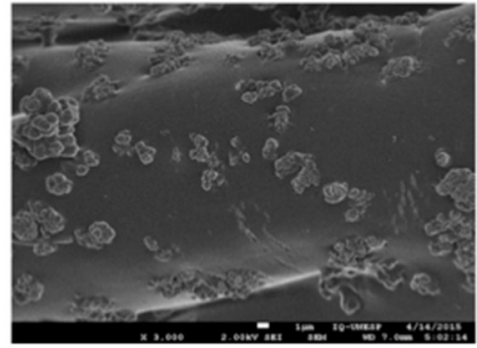

G

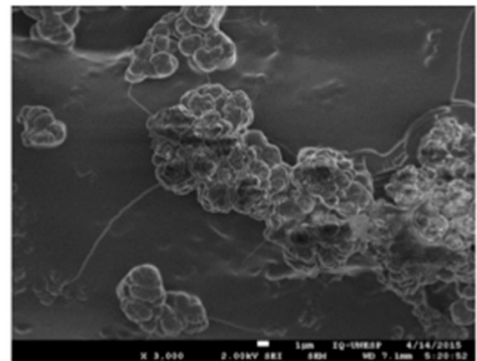

E

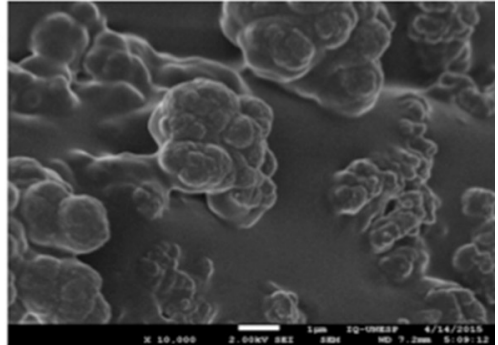

$\mathrm{H}$

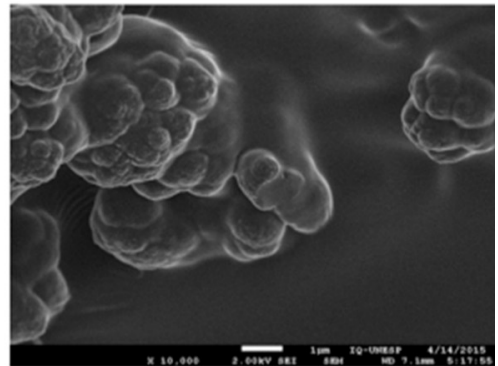

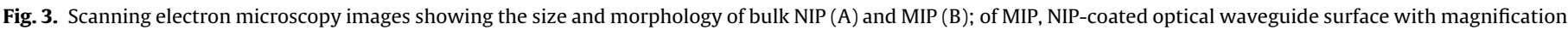
$100(C, F) ; 3000(D, G)$ and 10,000 (E, H), respectively.

violet 51 (DV51), Acid red 114 (AR114) and Direct Yellow 50 (DY50), commonly employed in the textile industry, were studied. The structures of the dyes together with their maximum absorption wavelengths are shown in Fig. 1. The calibration curves of all dyes are shown in Fig. S3, SI.

Stock solutions of $100 \mu \mathrm{M}$ of each dye were prepared in water and polymer particles, at a concentration of $20 \mathrm{mg} / \mathrm{mL}$, were suspended by sonication in water. From these stocks, polymer at a concentration of $8 \mathrm{mg} / \mathrm{mL}$ was pipetted in $1.5 \mathrm{~mL}$ polypropylene microcentrifuge tubes and $50 \mu \mathrm{M}$ of each dye was added to different tubes. The final volume was adjusted to $1 \mathrm{~mL}$ with water. The tubes were incubated for $4 \mathrm{~h}$ at ambient temperature on a tube rotator, followed by centrifugation for $30 \mathrm{~min}$ at $30,000 \mathrm{~g}$. Absorbance measurements at the wavelength (indicated in Fig. 1) of the dye analyzed were performed on the supernatants. The amount of dye bound to the polymers was calculated by subtracting the amount of unbound dye from the initial amount of dye added to the mixture.

\subsection{Dip-coating of polystyrene optical waveguides with MIP particles, and binding studies}

The waveguide was immersed into a glass vial containing $80 \mathrm{mg}$ of polymer and $240 \mathrm{mg}$ of polyvinyl alcohol (PVA) $\left(M_{\mathrm{w}}\right.$ :
$89,000-98,000)$ suspended in $4 \mathrm{~mL}$ of water. The mixture was heated at $90^{\circ} \mathrm{C}$ in a water-bath under gentle magnetic agitation. After $30 \mathrm{~min}$, the vial was allowed to cool to room temperature under gentle agitation. The fiber was then removed from the vial and dried in air, resulting in an immobilization of MIP particles on the fiber surface (Figs. 2A and $3 \mathrm{C}-\mathrm{H}$ ), held in place by the PVA acting as glue (insoluble in cold solvent). For binding studies, the MIP-coated optical fibers were placed in $4 \mathrm{~mL}$ glass vials containing solutions of $B R 9(0,5,10,15,20,25,30$ and $35 \mu \mathrm{M})$ in water $(10 \mathrm{~min}$ incubation).

After incubation, the bifurcated fiber optic probe of a spectrofluorimeter (Horiba Jobin-Yvon Fluorolog-3, Longjumeau, France) was used to inject visible light $(\lambda=545 \mathrm{~nm})$ through the MIP-coated fiber optic waveguide. The unabsorbed light was recorded through the same fiber optic probe (Fig. 2B). Measurements were performed with the MIP fiber remaining immersed into the analyte solution. The light intensity was monitored at $\lambda=545 \mathrm{~nm}$. The measured light intensity was converted into absorbance using

$A=-\log \left(I_{1} / I_{0}\right)$

where $A$ stands for absorbance, $I_{1}$ being the light intensity recorded in the presence of polymer and dye and $I_{0}$ being the light intensity recorded with the polymer alone. 

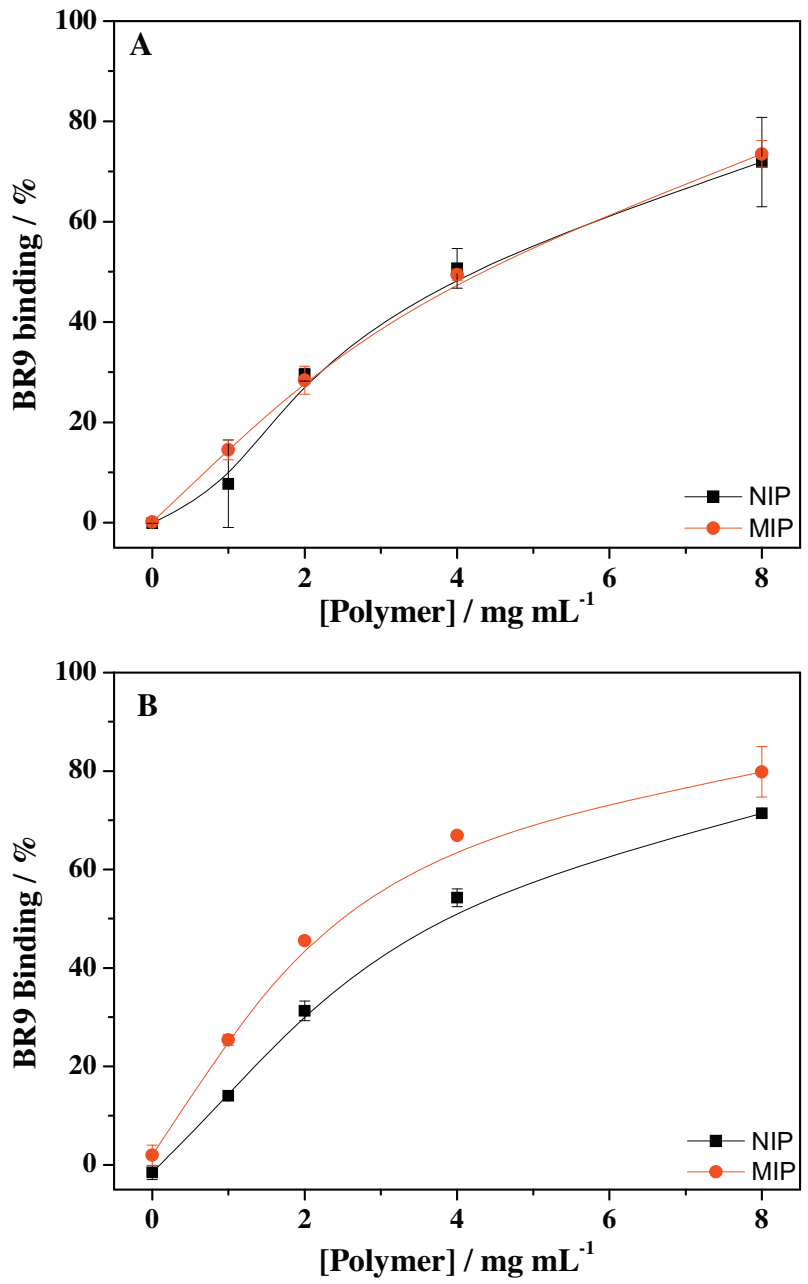

Fig. 4. Equilibrium binding isotherms of $10 \mu \mathrm{MBR}$ B with MIP(red) and NIP(black) in (A) water, (B) $1.0 \% \mathrm{NH}_{3 \text { (aq.) }}$. Data are means of duplicate independent experiments. (For interpretation of the references to color in this figure legend, the reader is referred to the web version of the article.)

\section{Results and discussions}

\subsection{Synthesis and evaluation of the binding behavior of the polymers}

The synthesis of MIPs for BR9 was inspired from the protocol described by Andersson and Ramström [26]. Their target molecule, 4,4'-diaminodiphenyl methane, a partial structural analogue of BR9 was imprinted using the functional monomer AMPSA which can make electrostatic interactions with the amino groups of the template. For our work, we brought a few modifications, especially chloroform was replaced by a mixture of methanol/water (4/1) to increase the chance of obtaining water-compatible MIPs [27]. An optimized ratio BR9:AMPSA:EGDMA of 1:3:200 was obtained, by varying the amount of cross-linkers [28,29]. The resultant polymers were characterized by scanning electron microscopy (SEM). As seen in Fig. 3A and B, the NIP and MIP particles have similar morphologies and sizes of $\sim 1 \mu \mathrm{m}$ which appear agglomerated. The same structures were seen in the three batches of synthesized polymers.

The binding behavior of the BR9 dye to the MIP was first evaluated in water ( $\mathrm{pH} \sim 6$ ). As seen in Fig. $4 \mathrm{~A}$, the polymers showed a high percentage of binding, but without specificity. Equilibrium binding assays were then performed at different pHs (2.0, 5.0, 7.0 and 10.0 ) by using Britton-Robinson buffer solution. At $\mathrm{pH}$ values of 2.0 and 10.0, the binding of BR9 to the polymers was found to

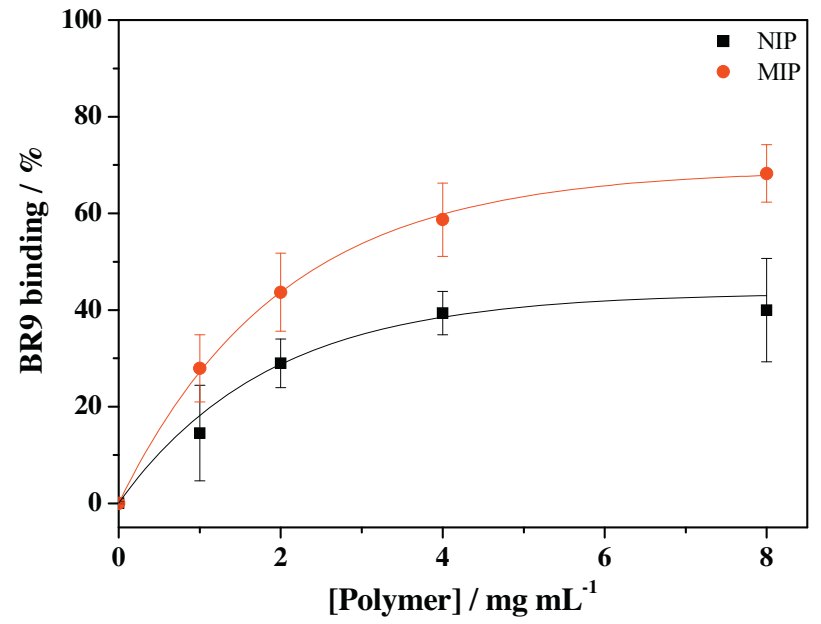

Fig. 5. Binding of $10 \mu \mathrm{M}$ BR9 to MIP and NIP in $0.05 \% \mathrm{NH}_{3(\text { aq.) }}$. Data are means from three independent experiments with three different batches of polymers. The error bars represent standard deviations.

present random and unexploitable results whereas at $\mathrm{pH} 5.0$ and 7.0 , the polymers presented a high binding but without specificity (results not shown). In an attempt to reduce non-specific interactions caused by the interaction of analyte with free reactive groups on the polymer surface, $1 \%(\mathrm{v} / \mathrm{v}) \mathrm{NH}_{3}$ solution was added. The ammonia molecule will compete with the dye for the free active groups on the polymeric structure hence favoring the binding of BR9 to specific cavities in the MIP. In the presence of $1 \% \mathrm{NH}_{3}$ solution, the binding specificity in water was improved (Fig. 4B). When a lower concentration of ammonia $(0.05 \%)$ was added, the specificity got still better (Fig. 5). An imprinting factor (IF), which corresponds to the ratio BR9 bound to the MIP versus BR9 bound to the NIP, of $\sim 1.6$ was obtained. Though low, this value was reproducible as similar binding profiles were observed with three independent batches of polymers. Concentrations of $\mathrm{NH}_{3}(0.025$ and $0.01 \%)$ produced no improvement of specificity (results not shown). Other attempts to improve the IF, like testing a series of dye concentrations while keeping a fixed concentration of polymer (Fig. S4, SI) or working at a fixed optimum concentration of dye and varying the amount of polymers (Fig. S5, SI) were studied, but without success.

Since BR9 is usually present with other dyes in textile effluents, selectivity studies were performed with six other textile dyes: Methyl Green (MG), Acid Green 16 (AG16), Acid Red 1 (AR1), Direct violet 51 (DV51), Acid red 114 (AR114) and Direct Yellow 50 (DY50) (Fig. 1). MG was chosen because it is a very close structural analogue of BR9 and most of the other dyes chosen were azo dyes as they represent the most important class of dyes in textile effluents [30]. $8 \mathrm{mg} / \mathrm{mL}$ of polymers were incubated with $50 \mu \mathrm{M}$ of each dye and the binding was compared. The dye concentration was chosen to be $50 \mu \mathrm{M}$ so as to have readable absorbance values of low-absorbing dyes while still working in the linear section of highly absorbing dyes (see calibration curves in Fig. S3, SI). Fig. 6 shows that BR9 MIP is quite selective, as the binding of BR9 was far more superior than other dyes in water. Noteworthy is the high selectivity, even towards the very close structural analogue, Methyl Green.

\subsection{Coating fiber optic waveguides with polymer particles and sensing of BR9}

The binding behavior of the MIP being characterized, we aimed to apply it for optical sensing by integration of the MIP into a fiber optic device. For this application, we used disposable 4-cm long injection-molded tapered polystyrene optical fibers $[17,19,20]$ (Fig. 2A). In our study, MIP particles were coated onto the fiber by 


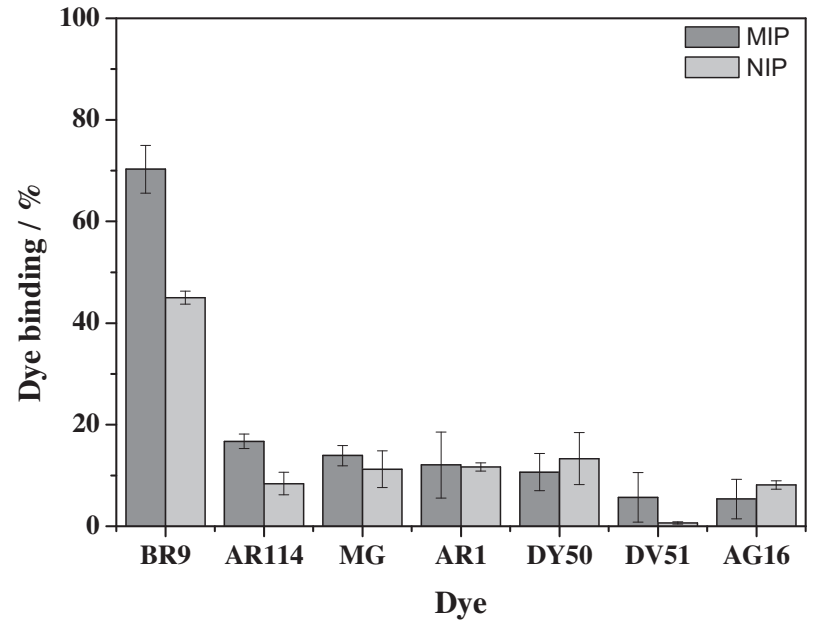

Fig. 6. Binding of different textile dyes $(50 \mu \mathrm{M})$ to BR9-templated MIP and NIP $(8 \mathrm{mg} / \mathrm{mL})$ in water. Absorbance was measured at the wavelength of the dye analyzed. Data are means from three independent experiments. The error bars represent standard deviations.
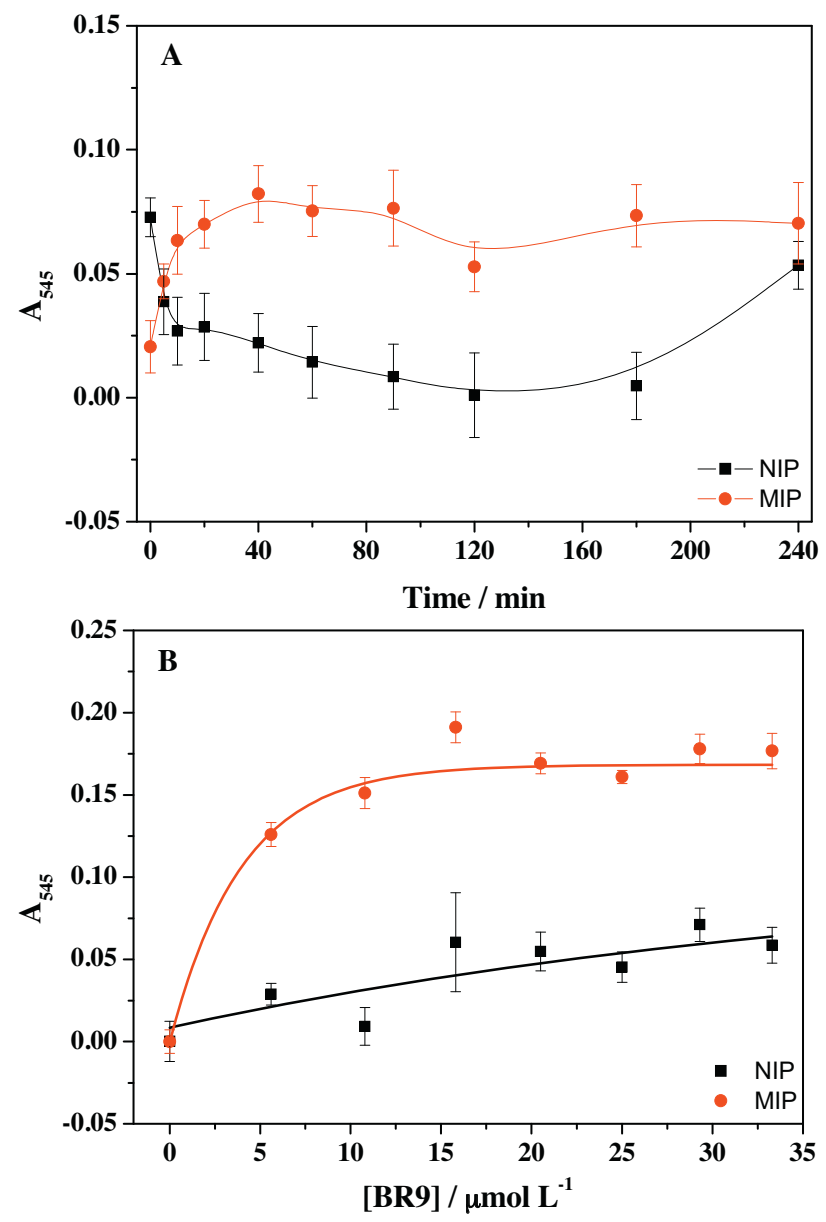

Fig. 7. Binding response of (A) $10 \mu \mathrm{M}$ BR9 with time ( $t$ ) and (B) with increasing concentrations of BR9, at $t=40 \mathrm{~min}$, after incubation of the polymer-coated optical fiber with BR9 in water. Data are means from three independent experiments with three different batches of polymers. The error bars represent standard deviations.

using PVA as glue [17,31]. PVA dissolves in hot water for the preparation of the polymer suspension, but after drying does not dissolve under the conditions of the assay. With our synthesis protocol, $\sim 5 \mathrm{~g}$ of polymer is obtained, enough to make about 60 immobilizations on the optical fiber. SEM images (Fig. 3C-H) prove the presence of the polymers on the fiber's surface.

Visible light was injected through the fiber optic bundle of the Horiba Fluorolog spectrofluorimeter and the resulting signal was collected via the same fiber optic probe, as illustrated in Fig. 2B. Binding analysis was performed by dipping the fiber in a series of dye concentrations in water, with the fiber remaining immersed in the analyte solution. The response time between the BR9 dye and the immobilized polymers on the optical fiber was first studied. As shown in Fig. 7A, the dye was already adsorbed to the MIP following 10 min of incubation; the maximum retention occurs after 40 min and the absorbance remains practically constant after this time. Therefore, $40 \mathrm{~min}$ of incubation for the binding of BR9 to the MIP was used in the subsequent steps.

The concentration-dependence of the MIP response upon binding to BR9 was then demonstrated, as well as the specificity of the MIP compared to the NIP (Fig. 7B). The maximum imprinting factor was $\sim 5$, higher than that reported with free polymers assayed in suspension (Fig. 5 and Figs. S4 and S5, SI). One explanation for the improvement of the binding specificity might lie in the accessibility of more binding cavities at the surface, since a thin layer of polymer was formed on the fiber. The MIP-coated fiber optic device could detect as low as $2 \mu \mathrm{M}$ of dye (Fig. 7B). Actually, there is no legislation for environmental dyes' limits but recent literature reports a tolerance of $\sim 1 \mathrm{mg} / \mathrm{L}$ for dyes coming from direct discharges of textile effluents in watercourses [30]. This amount corresponds to $3 \mu \mathrm{M}$ of BR9, indicating that the dye could eventually be detected by our sensor. However, to increase the performance of the sensor, more uniform-sized MIPs nanoparticles, for instance those synthesized by precipitation polymerization could be tested as they might generate a more homogeneous polymer coating on the fiber [17].

\section{Conclusions}

We synthesized a MIP for the textile dye BR9. Batch equilibrium binding studies of bulk polymers showed that the MIP was specific as it binds more BR9 than the NIP, in water. The BR9 MIP was also selective with binding $\sim 4$ to 13-fold higher for BR9 over other textile dyes, varying from very close structural analogues to nonrelated dyes. The MIP was immobilized on a disposable evanescent waveguide and detection of BR9 was achieved in the low $\mu \mathrm{M}$ range. Thus, our MIP for BR9 is really promising for the development of optical sensors for specific and sensitive determination of this compound in effluents. This initial proof-of-concept study of the detection of a model dye in water, will further pave the way to the development of this type of sensor for other dyes. Further work is in progress to evaluate their applications in industrial textile effluents.

\section{Acknowledgements}

The authors gratefully acknowledge financial supports from FAPESP (processes number: 2008/10449-7, 2011/17552-0 and 2013/02576-7), the European Regional Development Fund ERDF, the Regional Council of Picardy (projects CBI-PEM and co-funding of equipment under CPER 2007-2013).

\section{Appendix A. Supplementary data}

Supplementary data associated with this article can be found, in the online version, at http://dx.doi.org/10.1016/j.snb.2015.05.007

\section{References}

[1] C. Alexander, H.S. Andersson, L.I. Andersson, R.J. Ansell, N. Kirsch, I.A. Nicholls, J. O'Mahony, M.J. Whitcombe, Molecular imprinting science and technology: a 
survey of the literature for the years up to and including 2003, J. Mol. Recognit. 19 (2006) 106-180

[2] B. Tse Sum Bui, K. Haupt, Molecularly imprinted polymers: synthetic receptors in bioanalysis, Anal. Bioanal. Chem. 398 (2010) 2481-2492.

[3] K. Haupt, A.V. Linares, M. Bompart, B. Tse Sum Bui, Molecularly imprinted polymers, in: K. Haupt (Ed.), Topics in Current Chemistry, Springer, Berlin/Heidelberg, 2012, pp. 1-28.

[4] R. Narayanaswamy, S.M. Ng, Molecularly imprinted sensing receptors, in: S.W. Lee, T. Kunitake (Eds.), Handbook of Molecular Imprinting: Advanced Sensor Applications, Pan Stanford Publishing, 2012, pp. 63-116.

[5] M.J. Whitcombe, I. Chianella, L. Larcombe, S.A. Piletsky, J. Noble, R. Porter, A. Horgan, The rational development of molecularly imprinted polymer-based sensors for protein detection, Chem. Soc. Rev. 40 (2011) 1547-1571.

[6] X.A. Ton, B. Tse Sum Bui, M. Resmini, P. Bonomi, I. Dika, O. Soppera, K. Haupt, A versatile fluorescence fiber optic sensor based on in-situ polymerized molecularly imprinted microstructures, Angew. Chem. Int. Ed. 52 (2013) 8317-8321.

[7] K. Kotova, M. Hussain, G. Mustafá, P.A. Lieberzeit, MIP sensors on the way to biotech applications: targeting selectivity, Sens. Actuators B 189 (2013) 199-202.

[8] W. Wan, M. Biyikal, R. Wagner, B. Sellergren, K. Rurack, Fluorescent sensing microparticles that 'light-up' consisting of a silica core and a molecularly imprinted polymer (MIP) shell, Angew. Chem. Int. Ed. 52 (2013) 7023-7027.

[9] M.D. Marazuela, M.C. Moreno-Bondi, Fiber-optic biosensors - an overview, Anal. Bioanal. Chem. 372 (2002) 664-682.

[10] G. Orellana, D. Haigh, New trends in fiber-optic chemical and biological sensors, Curr. Anal. Chem. 4 (2008) 273-295.

[11] X.D. Wang, O.S. Wolfbeis, Fiber-optic chemical sensors and biosensors (2008-2012), Anal. Chem. 85 (2013) 487-508.

[12] X.Q. Yang, X.X. Zhao, C.Y. Liu, Y. Zheng, S.J. Qian, Decolorization of azo, triphenylmethane and anthraquinone dyes by a newly isolated Trametes sp. SQ01 and its laccase, Process Biochem. 44 (2009) 1185-1189.

[13] C.C.I. Guaratini, M.V.B. Zanoni, Corantes têxteis, Quím. Nova 23 (2000) 71-78.

[14] A.B. Santos, F.J. Cervantes, J.B. Van Lier, Review paper on current technologies for decolourisation of textile wastewaters: perspectives for anaerobic biotechnology, Bioresour. Technol. 98 (2007) 2369-2385.

[15] X. Yang, J. Wang, X. Zhao, Q. Wang, R. Xue, Increasing manganese peroxidase production and biodecolorization of triphenylmethane dyes by novel fungal consortium, Bioresour. Technol. 102 (2011) 10535-10541.

[16] L. Ayed, K. Chaieb, A. Cheref, A. Bakhrou, Biodegradation and decolorization of triphenylmethane dyes by Staphylococcus epidermidis, Desalination 260 (2010) $137-146$

[17] X.A. Ton, V. Acha, P. Bonomi, B. Tse Sum Bui, K. Haupt, A disposable evanescent wave fiber optic sensor coated with a molecularly imprinted polymer as a selective fluorescence probe, Biosens. Bioelectron. 64 (2015) 359-366.

[18] C.R. Taitt, G.P. Anderson, F.S. Ligler, Evanescent wave fluorescence biosensors, Biosens. Bioelectron. 20 (2005) 2470-2487.

[19] D.V. Lim, Detection of microorganisms and toxins with evanescent wave fiberoptic biosensors, Proc. IEEE 91 (2003) 902-907.

[20] G.P.Anderson, K.D. King, K.L. Gaffney, L.H. Johnson, Multi-analyte interrogation using the fiber optic biosensor, Biosens. Bioelectron. 14 (2000) 771-777.

[21] R. Narayanaswamy, F. Sevilla III, Absorbance spectroscopy for chemical sensors, in: J.H. Steele, K.K. Turekian, S.A. Thorpe (Eds.), Encyclopedia of Ocean Sciences, Elsevier Ltd, 2008, pp. 3407-3415.

[22] Y. Mendelson, Optical Sensors, in: J.D. Bronzino (Ed.), The Biomedical Engineering Handbook, second ed., CRC Press LLC, 2000 (Chapter 50).

[23] Basic Red 9 Monohydrochloride: CAS No. 569-61-9, in: Report on Carcinogens, 12th edition, National Toxicology Program, Department of Health and Human Services, 2011.

[24] A.O. Martins, V.M. Canalli, C.M.N. Azevedo, M. Pires, Degradation of pararosaniline (C.I. Basic Red 9 monohydrochloride) dye by ozonation and sonolysis, Dyes Pigments 68 (2006) 227-234.

[25] National Toxicology Program, Toxicology and carcinogenesis studies of C.I. basic red 9 monohydrochloride (pararosaniline) (CASNo. 569-61-9) in F344/N rats and B6C3F1 mice (feed studies), Natl. Toxicol. Program Tech. Rep. Ser. 285 (1998) $1-228$.

[26] H.S. Andersson, O. Ramström, Crown ethers as a tool for the preparation of molecularly imprinted polymers, J. Mol. Recognit. 11 (1998) 103-106.

[27] K. Haupt, A. Dzgoev, K. Mosbach, Assay system for the herbicide 2,4dichlorophenoxyacetic acid using a molecularly imprinted polymer as an artificial recognition element, Anal. Chem. 70 (1998) 628-632.
[28] Z. Zhang, H. Li, H. Liao, L. Nie, S. Yao, Influence of cross-linkers' amount on the performance of the piezoelectric sensor modified with molecularly imprinted polymers, Sens. Actuators B 105 (2005) 176-182.

[29] E. Yilmaz, K. Mosbach, K. Haupt, Influence of functional and cross-linking monomers and the amount of template on the performance of molecularly imprinted polymers in binding assays, Anal. Commun. 36 (1999) 167-170.

[30] C. Zaharia, D. Suteu, Textile organic dyes - characteristics, polluting effects and separation/elimination procedures from industrial effluents - a critical overview, in: T. Puzyn (Ed.), Organic Pollutants Ten Years After the STOCKHOLM Convention - Environmental and Analytical Update, InTech, 2012 (available from http://www.intechopen.com/books/authors/organicpollutants-ten-years-after-the-stockholm-convention-environmental-andanalytical-update/textile-organic-dyes-characteristics-polluting-effects-andseparation-elimination-procedures-from-in).

[31] I. Surugiu, B. Danielsson, L. Ye, K. Mosbach, K. Haupt, Chemiluminescence imaging ELISA using an imprinted polymer as the recognition element instead of an antibody, Anal. Chem. 73 (2001) 487-491.

\section{Biographies}

Marcos Vinicius Foguel received his Bachelor degree (2008) and Master degree in Chemistry (2011) from the Institute of Chemistry of Universidade Estadual Paulista (UNESP) in Araraquara, São Paulo (Brazil). During his Master's tenure, he worked in the development of impedimetric sensor for aflatoxin $B_{1}$ in food samples in collaboration with Masarik University (Brno, Czech Republic). His broad research interests lie in the development of electrochemical and optical sensors. Currently, he is working on the development of optical sensors for the detection of dyes at Universidade Estadual Paulista (UNESP) as a PhD student, in collaboration with Compiègne University of Technology (UTC - France).

Xuan-Anh Ton studied Chemistry at the Industrial Physics and Chemistry Higher Educational Institution (ESPCI ParisTech), in Paris (France), where she graduated in 2010 with a degree of Chemical Engineering (eq. MSc). In 2013, she completed her $\mathrm{PhD}$ in Biotechnology at Compiègne University of Technology (France), in the group of Prof. Karsten Haupt. She is currently a research fellow at Max Planck Institute for Polymer Research (Mainz, Germany), and her current research interests include biomimetic polymers and tissue engineering.

Maria Valnice Boldrin Zanoni graduated from the Institute of Chemistry of São Paulo University (USP) with PhD in Physical Chemistry, in 1989. Her current academic position is at the University of São Paulo (UNESP), Brazil, as a full professor in Analytical Chemistry. Her main research interests are electroanalysis and environmental electrochemistry, investigating analytical methodologies and new treatment methods of dyes. She expanded her research interests to electrochemical sensors applied to dye and pharmaceutical compounds detection.

Maria Del Pilar Taboada Sotomayor studied Chemistry at the Universidad Nacional de Ingenieria (Lima, Peru) from 1987 to 1992, where she obtained her BSc degree in Chemistry. In 1996 she obtained her MSc degree in Analytical Chemistry at UNICAMP (Brazil), and in March 2000 received her PhD in Sciences at UNICAMP. She currently lectures at the Institute of Chemistry of UNESP in Araraquara, São Paulo (Brazil). She works mainly in the development of electrochemical and optical biomimetic sensors based on biomimetic catalysts and molecularly imprinted polymers.

Karsten Haupt studied Biochemistry at the University of Leipzig, Germany. In 1994 he obtained his PhD in Bioengineering from Compiègne University of Technology, France. He then held research positions at Lund University, Sweden, and at INSERM, Paris, France, before joining the University of Paris 12 as an associate professor in 1999. In 2003 he was appointed full professor of Nanobiotechnology at Compiègne University of Technology, France, where he is now the Head of the CNRS Institute for Enzyme and Cell Engineering. His present research interests include affinity technology, chemical and biosensors, molecularly imprinted polymers and synthetic receptors, and nanostructured materials for biomedical applications.

Bernadette Tse Sum Bui obtained her MSc in Chemistry at the University of AixMarseille and her PhD in Biotechnology at Compiègne University of Technology (France). After working 13 years as a CNRS research engineer in Biochemistry at the University Pierre and Marie Curie, Paris, she joined Pr. Karsten Haupt's group in 2006 to work on molecularly imprinted polymers and their applications in separation, drug delivery and sensors. 